Proceedings - 13th International Conference on Signal-Image Technology and InternetBased Systems, SITIS 2017Volume 2018-January, 9 April 2018, Pages 35-3913th International Conference on Signal-Image Technology and Internet-Based Systems, SITIS 2017; Jaipur; India; 4 December 2017 through 7 December 2017; Category numberE6332; Code 135826

\title{
Implementation and customization of a smart mirror through a facial recognition authentication and a personalized news recommendation algorithm(Conference Paper)
}

- Garcia, I.C.A.Email Author,

- Salmon, E.R.L.Email Author,

- Riega, R.V.Email Author,

- Padilla, A.B.Email Author

- Computer Science, Universidad Peruana de Ciencias Aplicadas, Lima, Peru

\begin{abstract}
View references (29)
In recent years the advancement of technologies of information and communication (technology ICTs) have helped to improve the quality of people's lives. The paradigm of internet of things (IoT, Internet of things) presents innovative solutions that are changing the style of life of the people. Because of this proposes the implementation of a smart mirror as part of a system of home automation, with which we intend to optimize the time of people as they prepare to start their day. This device is constructed from a reflective glass, LCD monitor, a Raspberry Pi 3, a camera and a platform IoT oriented cloud computing, where the information is obtained to show in the mirror, through the consumption of web services. The information is customizable thanks to a mobile application, which in turn allows the user photos to access the mirror, using authentication with facial recognition and user information to predict the news to show according to your profile. In addition, as part of the idea of providing the user a personalized experience, the Smart Mirror incorporates a news recommendation algorithm, implemented using a predictive model, which uses the algorithm, naive bayes. () 2017 IEEE.
\end{abstract}

\section{SciVal Topic Prominence}

Topic: $\underline{\text { Cameras } \mid \text { Computer vision | egocentric video }}$

Prominence percentile: 94.355

\section{Author keywords}

MagicMirrorRecognitionRecommendationSmart

- ISBN: 978-153864283-2

- Source Type: Conference Proceeding 
- Original language: English

- DOI: $10.1109 /$ SITIS.2017.17

- Document Type: Conference Paper

- Volume Editors: Dipanda A.,Chbeir R.,Nain N.,Yetongnon K.,Gallo L.

- Sponsors: Department of Science and Technology Government of Rajasthan,Institute of High Performance Computing and Networking (ICAR) of the National Research Center of Italy,Laboratoire Electronique, Image et Informatique (LE2I),Malaviya National Institute of Technology Jaipur (MNIT),University of Bourgogne,University of Milan

- Publisher: Institute of Electrical and Electronics Engineers Inc. 